\title{
Efeito do flushing sobre o desempenho reprodutivo de ovelhas Morada Nova e Santa Inês submetidas à inseminação artificial em tempo fixo
}

\author{
[The effect of flushing on the reproductive performance of Morada Nova and Santa Inês ewes \\ submitted to fixed time artificial insemination] \\ F.P. Gottardi ${ }^{1,2}$, A. Souza Júnior ${ }^{3}$, Y.G.S. Barbosa ${ }^{2}$, C.A.T. Marques ${ }^{2}$, L.R. Bezerra ${ }^{2}$, \\ M.J. Araújo ${ }^{2}$, G.Z. Mingoti ${ }^{1,4}$, J.N.C. Torreão ${ }^{2}$ \\ ${ }^{1}$ Universidade Estadual Paulista - FCAV-UNESP - Jaboticabal, SP \\ ${ }^{2}$ Universidade Federal do Piauí - CPCE - Bom Jesus, PI \\ ${ }^{3}$ Universidade Federal do Piauí - CTT - Teresina, PI \\ ${ }^{4}$ Universidade Estadual Paulista - FCAV-UNESP - Araçatuba, SP
}

\begin{abstract}
RESUMO
O objetivo do estudo foi analisar o efeito do flushing, do protocolo hormonal para sincronização de estro e da IATF sobre o desempenho reprodutivo de ovelhas Morada Nova (MN) e Santa Inês (SI). Foram utilizadas 27 ovelhas SI e 24 ovelhas MN suplementadas com concentrado (1\% do peso vivo, em média) durante 75 dias de estação reprodutiva. Após 30 dias de suplementação, as ovelhas foram sincronizadas com protocolo hormonal $(\mathrm{PH})$ à base de progestágeno, eCG e cloprostenol. A observação de estro foi realizada após 12, 24, 36 e 48 horas do término do PH, com o auxílio de dois rufiões, e a IATF foi realizada aproximadamente 55 horas após o final do $\mathrm{PH}$. Do $20^{\circ}$ ao $45^{\circ}$ dia após o início do $\mathrm{PH}$, realizouse o repasse com um reproduto Dorper. O diagnóstico de prenhez foi realizado 70 dias após a IATF. Foi analisado o peso, escore da condição corporal (ECC), taxa de apresentação de estro, taxa de prenhez e prolificidade, testando-se os efeitos da raça, semana de suplementação (SS) e classe de ECC. O peso e ECC das ovelhas variaram em função da SS. Foi observada taxa de estro de $88,2 \%$, sendo que $43,2 \%$ das ovelhas apresentaram estro até 24 horas do final do PH. A taxa de prenhez por IATF foi de $31,4 \%$ e a de prenhez após repasse foi de 50,0\%, sendo que a classe de ECC interferiu nas taxas de prenhez. Obteve-se maior porcentagem de partos múltiplos de ovelhas prenhes por IATF do que por monta natural no repasse. Conclui-se que o flushing resultou em ganho de peso e aumento do ECC, o que garantiu a padronização do rebanho para a estação reprodutiva e, consequentemente, melhorou o desempenho reprodutivo. O PH utilizado aumentou a prolificidade e adiantou o início do estro; porém, não foi eficiente na sincronização de ovelhas deslanadas.
\end{abstract}

Palavras-chave: ovino, condição corporal, estro, prolificidade, sincronização

\begin{abstract}
The aim of this study was to analyze the effect of flushing on the reproductive performance of Morada Nova (MN) and Santa Inês (SI) ewes submitted to fixed time artificial insemination (TAI). Twenty seven SI and 24 MN supplemented with concentrate (1\% of live weight, on average), for 75 days during the breeding season. After 30 days of supplementation, ewes were synchronized with the aid of a hormonal protocol (HP) based on progesterone, eCG and cloprostenol. The estrus observation was conducted at 12, 24, 36 and $48 \mathrm{~h}$ after the end of HP with the aid of two ruffians. TAI was done $55 \mathrm{~h}$ after the end of HP. From 20 to 45 days after the beginning of the HP ewes were exposed to rams (natural breeding). The pregnancy diagnosis was evaluated 70 days after TAI. We analyzed the weight, body condition score, estrus rate, pregnancy rate and prolificacy testing the effects of race, week of supplementation and body condition score class. The weight and body conditions of ewes varied according to the week of supplementation, with higher values in the first two weeks following TAI. The estrus rate was $88.2 \%$ and
\end{abstract}

Recebido em 24 de agosto de 2012

Aceito em 7 de novembro de 2013

E-mail: fpgottardi@ufpi.edu.br 
43.2\% of the ewes showed estrus up to 24 hours of the end of the HP. The pregnancy rate per TAI was $31.3 \%$ and the pregnancy rate after natural breeding was $50.0 \%$. It was observed that body condition score classes interfered in pregnancy rates. There was a higher percentage of multiple births by pregnancy by TAI than by natural breeding. It was concluded that the flushing resulted in weight gain and better body conditions ensuring the standardization of the herd for breeding season, which therefore improved reproductive performance. The HP used advanced the onset of estrus and increased prolificacy, but was inefficient in the synchronization of woolless sheep.

Keywords: ovine, body conditions, estrus, litter size, synchronization

\section{INTRODUÇÃO}

No Brasil, a criação e o mercado de carne de ovinos estão em amplo crescimento, assumindo um papel relevante na economia do nordeste do país, onde estão localizados $56,7 \%$ dos animais do país, sendo a maioria dos rebanhos compostos por ovinos deslanados da raça Morada Nova e Santa Inês (Anuário da pecuária brasileira, 2012).

As ovelhas deslanadas são caracterizadas por serem animais poliéstricos e adaptados às regiões semiáridas; no entanto, ainda apresentam resultados insatisfatórios de desempenho reprodutivo, ocasionado, dentre outros fatores, pela variação da condição corporal individual dos animais ao longo da estação reprodutiva, que é resultante da escassez de alimento em determinadas épocas do ano e a falta de estrutura técnica do produtor (Catunda, 2011).

Para melhorar essa condição, os esforços devem se concentrar no aumento da produtividade no semiárido nordestino com investimentos em técnicas nutricionais e reprodutivas.

A utilização de suplementação alimentar em torno de quatro semanas antes e quatro semanas após a concepção (flushing) melhora a condição corporal das ovelhas e, consequentemente, aumenta a taxa de concepção, de ovulação e a sobrevivência embrionária (Boucinhas et al., 2006).

No entanto, o flushing deve ser bem empregado, pois tanto a subnutrição como a supernutrição contribuem para as perdas reprodutivas (Ribeiro, 2003). Desse modo, a suplementação é preconizada para fêmeas com ECC entre 2,0 e 2,5; de forma que, ao serem submetidas à suplementação iniciem a estação de acasalamento ganhando peso, atingindo ECC entre 3,0 e 3,5 (Ribeiro, 2003).

Em relação às técnicas reprodutivas, a utilização de hormônios específicos para sincronizar o estro e ovulação, aliados às biotecnologias, como a inseminação artificial em tempo fixo (IATF), tende a melhorar o desempenho reprodutivo por aumentar o efeito do melhoramento genético de elevado valor genético e facilitar o manejo reprodutivo (Catunda, 2011; Padilha, 2011).

Os protocolos de sincronização de estro têm boa eficiência para ovinos, porém a técnica de IATF apresenta entraves para a difusão em rebanhos comerciais, principalmente pela dificuldade técnica da transposição do canal cervical para deposição intrauterina do sêmen. Devido a isso, tem sido observada grande variabilidade de resultados em programas de sincronização de ovulação, estro e IATF (Bicudo, 2003).

Qualquer que seja a técnica de inseminação, a determinação do momento da ovulação e da realização da IATF é crucial para o sucesso. Menchaca e Rubianes (2004) descrevem que resultados satisfatórios de taxa de prenhez são obtidos quando as inseminações intracervicais são feitas entre 54 e 60 horas da retirada da esponja de progestágeno.

Para a utilização de flushing e IATF, e proposição de um manejo reprodutivo eficiente, torna-se necessária a realização de estudos que avaliem o efeito de suplementos alimentares e hormônios exógenos sobre a fisiologia reprodutiva de ovinos explorados na região nordeste. Dessa forma, o objetivo do trabalho foi analisar o efeito do flushing, do protocolo hormonal de sincronização de estro e IATF sobre o desempenho reprodutivo de ovelhas da raça MN e SI criadas a pasto no sul do Estado do Piauí. 


\section{MATERIAL E MÉTODOS}

O estudo foi realizado durante o período de setembro a novembro de 2011, no setor de pesquisa de pequenos ruminantes do Colégio Técnico da cidade de Bom Jesus, Piauí, localizada a $09^{\circ} 04^{\prime}$ de latitude sul, $44^{\circ} 21^{\prime}$ de longitude oeste e $277 \mathrm{~m}$ de altitude.

O clima da região é classificado como subúmido seco (Andrade Júnior, 2005), com temperaturas mínimas de $18^{\circ} \mathrm{C}$ e máximas de $36^{\circ} \mathrm{C}$. A precipitação pluviométrica média anual é definida no Regime Equatorial Continental, com precipitações anuais em torno de 800 a $1.200 \mathrm{~mm}$ e período chuvoso estendendo-se de dezembro a maio.

A utilização de animais para o experimento foi aprovada pelo comitê de ética em experimentação com animais CEEA/UFPI sob o protocolo de número 091/2010. Foram utilizadas 51 ovelhas entre 3 e 6 anos de idade, pluríparas, não gestantes, sadias, vermifugadas e vacinadas contra enterotoxemia, sendo 27 animais da raça Santa Inês (SI) com peso médio de 43,7 kg e 24 da raça Morada Nova (MN) com peso médio de $25,7 \mathrm{~kg}$. As fêmeas foram selecionadas pelo histórico reprodutivo e por ultrassonografia abdominal para avaliação do útero, sendo descartadas as ovelhas prenhes ou com patologias uterinas.

Durante os 75 dias de estação reprodutiva, as fêmeas permaneceram em piquetes de lotação alternada com pastagem de Andropogon gayanus durante o dia. Ao final da tarde eram recolhidas para baias coletivas, onde passavam a noite e recebiam nos comedouros suplementação a $1 \%$ do peso vivo dos animais, tendo como base o preconizado pelo NRC (Nutrient..., 2007). A ração concentrada fornecida foi formulada à base de farelo de milho, farelo de soja e suplemento vitamínico e mineral, contendo 90,45\% de MS, $75 \%$ de NDT, $13 \%$ de PB e $9,34 \%$ de FB.

Foram mensurados semanalmente o peso e o escore da condição corporal (ECC) das ovelhas MN e SI. O ECC foi determinado segundo metodologia descrita por Thompson e Meyer (1994), atribuindo-se valores de 1 a 5 , em que 1 corresponde a animais muito magros e 5, a animais obesos.

O protocolo de indução e sincronização do estro foi realizado com esponjas vaginais $(60 \mathrm{mg}$ de acetato de medroxiprogesterona: Progespon ${ }^{\circledR}$ Syntex S.A, Argentina), as quais permaneceram por nove dias na porção cranial da vagina, sendo o dia de aplicação da esponja considerado como dia zero do protocolo hormonal (D0). No dia da retirada da esponja (dia 9) foram administrados, em cada animal, por via intramuscular, 200UI de eCG (Folligon 5000®, Intervet, Holanda) e 0,1 mg de cloprostenol sódico (Ciosin ${ }^{\circ}$, Intervet, Holanda). A observação visual do estro foi realizada por no mínimo 30 minutos, com auxílio de dois rufiões colocados juntos com as fêmeas após 12, 24, 36 e 48 horas da retirada da esponja.

O sêmen utilizado para IATF foi obtido de dois reprodutores Dorper. Foi colhido com auxílio de vagina artificial e, em seguida, avaliado quanto à motilidade, vigor e concentração. Após avaliação, foi diluído em solução composta por água de coco, citrato de sódio a $5 \%$ e água destilada (na proporção $2: 1: 1$, respectivamente), para obtenção de concentração final de $3 \times 10^{6}$ espermatozoides por $\mathrm{mL}$ em palhetas de $0,25 \mathrm{~mL}$. Para cada ovelha foi realizada uma inseminação via transcervical com sêmen a fresco, em torno de 55 horas após a retirada da esponja intravaginal e administração dos hormônios.

Foi realizado o repasse com identificação do retorno ao estro e monta natural, utilizando-se um reprodutor da raça Dorper. $\mathrm{O}$ reprodutor foi colocado com as fêmeas diariamente às $7 \mathrm{e}$ às 18 horas, por no mínimo quarenta minutos, do $20^{\circ}$ ao $45^{\circ}$ dia do início da sincronização.

Para determinação da prenhez, foi realizada ultrassonografia abdominal, com um equipamento Chisson (Mod. 6600, China; transdutor linear 5,0 Mhz), 70 dias após a IATF. $\mathrm{O}$ diagnóstico de prenhez positivo resultante da IATF foi considerado para aquelas ovelhas que não apresentaram estro durante $\mathrm{o}$ repasse $\mathrm{e}$ confirmado pela data do parto, considerando tempo de gestação de 150 a 160 dias.

O delineamento do experimento está representado na Figura 1. 


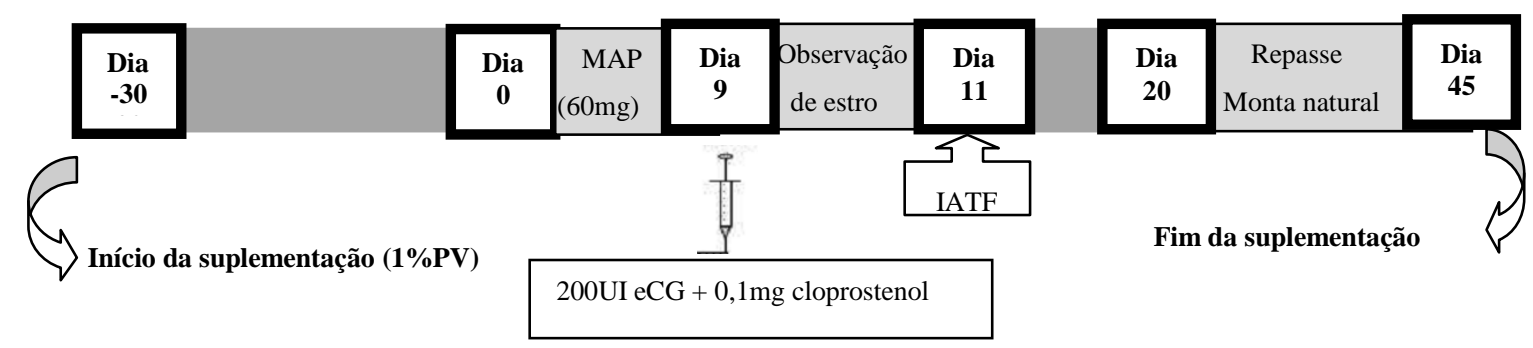

Figura 1. Delineamento experimental da estação reprodutiva de ovelhas da raça Morada Nova e Santa Inês.

Utilizou-se um delineamento inteiramente ao acaso (DIC), em que foram analisados os seguintes parâmetros: peso e escore da condição corporal (ECC) médio durante as semanas de suplementação; momento (horas) do início do estro após o final do protocolo hormonal para indução de estro; taxa de manifestação de estro após o final do protocolo hormonal $\left(\mathrm{n}^{\circ}\right.$ de fêmeas que manifestaram estro/ $\mathrm{n}^{\circ}$ de fêmeas expostas ao protocolo hormonal x 100); taxa de manifestação do estro durante o repasse $\left(\mathrm{n}^{\circ}\right.$ de fêmeas que manifestaram estro após IATF/ $\mathrm{n}^{\circ}$ de fêmeas expostas ao protocolo hormonal x 100); taxa de prenhez resultante da IATF $\left(\mathrm{n}^{\mathrm{o}}\right.$ de fêmeas prenhes por IATF $/ n^{\circ}$ de fêmeas expostas a IATF $\mathrm{x}$ 100); taxa de prenhez resultante de monta natural durante o repasse $\left(\mathrm{n}^{\mathrm{o}}\right.$ de fêmeas prenhes após monta natural/ $\mathrm{n}^{\circ}$ de fêmeas não prenhes por IATF x 100); e taxa de prenhez total da estação reprodutiva $\left(\mathrm{n}^{\mathrm{o}}\right.$ de fêmeas prenhes da IATF $+n^{\circ}$ de fêmeas prenhes após repasse/ $n^{\circ}$ de fêmeas utilizadas na estação reprodutiva x100).

Os dados referentes ao peso e ECC foram analisados pelo procedimento GLM do programa estatístico do SAS para verificação do efeito da raça, semana de suplementação, classe de ECC $(\leq 2 ;>2 \leq 3 ;>3)$ e interações. As médias dos efeitos significativos foram comparadas pelo teste de Tukey a 5\% de probabilidade. Foi realizada correlação simples de Pearson entre peso e ECC. As taxas de estro e prenhez foram comparadas entre as raças e classe de escore da condição corporal pelo teste de Fisher a 5\% de probabilidade. O tipo de parto (simples ou múltiplo) foi comparado pelo mesmo teste entre prenhez resultante da IATF e prenhez por monta natural durante o repasse.

\section{RESULTADOS E DISCUSSÃO}

O peso corporal das ovelhas durante a estação reprodutiva variou em função da raça, semana de suplementação e classe de escore da condição corporal $(\mathrm{Pr}>\mathrm{F}=0,01)$, sem efeito da interação entre raça e semana de suplementação $(\mathrm{Pr}>\mathrm{F}=0,05)$.

$\mathrm{O}$ peso médio corporal das ovelhas da raça $\mathrm{MN}$ foi menor do que o peso médio das fêmeas SI ( $<<0,05$, Tab. 1). O peso corporal em ovinos é variável com a raça, presença ou ausência de lã, estado gestacional e conteúdo gastrointestinal (Nutrient..., 2007). No caso do presente estudo, o fator raça foi o responsável, já que ovelhas MN apresentam porte menor do que ovelhas SI.

As ovelhas de ambas as raças (SI e MN) apresentaram maior peso médio na quinta $(38,84 \pm 1,62 \mathrm{~kg})$ e sexta $(38,80 \pm 1,55 \mathrm{~kg})$ semanas de suplementação (Tab. 1), o que demonstra o efeito do flushing. Resultados semelhantes foram relatados por Catunda (2011), que verificou um maior peso em ovelhas MN e SI após 4 e 5 semanas de flushing. A variação do ECC durante as semanas de suplementação foi semelhante à do peso, com maior ECC médio das ovelhas na quinta $(2,94 \pm 0,08)$ e sexta $(2,80 \pm 0,06)$ semanas de suplementação (Tab.1). Essa semelhança indica uma associação entre peso e ECC, a qual pôde ser comprovada pela correlação de Pearson, cujo coeficiente de correlação foi de 0,42 $(\mathrm{p}<0,01)$ para ovelhas da raça $\mathrm{MN}$ e de 0,58 $(\mathrm{p}<0,01)$ para ovelhas da raça SI. Outros autores demonstraram a relação peso e ECC para raças ovinas (Mori et al., 2006; Catunda, 2011), comprovando a eficiência da avaliação do ECC para estimar o estado nutricional de ovelhas deslanadas. 
Tabela 1. Médias ajustadas e erro padrão da média do peso $(\mathrm{kg})$ e escore da condição corporal (ECC) de ovelhas Morada Nova (MN) e Santa Inês (SI) em função dos efeitos da raça, semana de suplementação (SS) e classe de ECC durante a estação reprodutiva

\begin{tabular}{ccc}
\hline Efeitos & Peso $(\mathrm{kg})$ & ECC \\
\hline Raça & & \\
MN & $27,7 \pm 0,8 \mathrm{~b}$ & $2,7 \pm 0,1 \mathrm{a}$ \\
SI & $46,8 \pm 1,7 \mathrm{a}$ & $2,7 \pm 0,1 \mathrm{a}$ \\
\hline F & $958,4^{* *}$ & 0,2 \\
\hline SS & & \\
$1^{\mathrm{a}}$ & $34,4 \pm 1,5 \mathrm{~b}$ & $2,5 \pm 0,1 \mathrm{~b}$ \\
$2^{\mathrm{a}}$ & $36,3 \pm 1,6 \mathrm{ab}$ & $2,7 \pm 0,1 \mathrm{ab}$ \\
$3^{\mathrm{a}}$ & $37,8 \pm 1,7 \mathrm{ab}$ & $2,7 \pm 0,1 \mathrm{ab}$ \\
$4^{\mathrm{a}}$ & $38,1 \pm 1,7 \mathrm{ab}$ & $2,8 \pm 0,1 \mathrm{ab}$ \\
$5^{\mathrm{a}}$ & $38,8 \pm 1,6 \mathrm{a}$ & $2,9 \pm 0,1 \mathrm{a}$ \\
$6^{\mathrm{a}}$ & $38,8 \pm 1,5 \mathrm{a}$ & $2,8 \pm 0,1 \mathrm{a}$ \\
$7^{\mathrm{a}}$ & $37,7 \pm 1,7 \mathrm{ab}$ & $2,7 \pm 0,1 \mathrm{ab}$ \\
$8^{\mathrm{a}}$ & $37,7 \pm 1,6 \mathrm{ab}$ & $2,7 \pm 0,1 \mathrm{ab}$ \\
\hline F & $3,5^{* *}$ & $4,3 * *$ \\
\hline Classe de & & \\
ECC & & \\
$\leq 2,0$ & $36,0 \pm 2,0 \mathrm{c}$ & - \\
$>2,0 \leq 3,0$ & $39,9 \pm 0,6 \mathrm{~b}$ & - \\
$>3,0$ & $44,6 \pm 1,6 \mathrm{a}$ & - \\
\hline F & $38,9^{* *}$ & - \\
\hline CV & 16,4 & 17,7 \\
\hline
\end{tabular}

Semana da sincronização de estro e IATF; **Pr>F= 0,001; CV=coeficiente de variação. Médias seguidas de mesma letra na coluna, para o mesmo efeito, não diferem (teste Tukey, 5\%).

No presente trabalho, observou-se maior número de animais com ECC entre 2,5 e 3,0 no momento da IATF do que no início da suplementação $(66,7 \%$ e $24,5 \%$, respectivamente). Esse resultado demonstra que a realização de suplementação alimentar durante a estação reprodutiva (flushing) levou ao aumento significativo de peso dos animais, com consequente melhora no ECC (Tab. 1 e Fig. 2) e padronização do ECC das ovelhas no momento da IATF (Fig. 2).

O ECC reflete a quantidade de tecido muscular e adiposo armazenado no corpo do animal em determinado momento do seu ciclo produtivo/reprodutivo (Cezar e Sousa, 2006). A condição corporal das ovelhas antes, durante e logo após o acasalamento influencia na porcentagem de prenhez e no número de cordeiros nascidos no rebanho (Ribeiro et al., 2003; Viñoles, 2005). Devido a isso, o ganho de peso e a melhora do ECC podem melhorar o desempenho reprodutivo dessas ovelhas e, por essa razão, o ECC é considerado uma ótima ferramenta para avaliação dos animais durante o manejo reprodutivo (Cezar e Sousa, 2006).

Deve-se considerar que ECC muito baixo ou muito alto no início do período de monta são indesejados. Por outro lado, flutuações decorrentes do aporte de energia, com aumento do ECC durante a estação reprodutiva, têm efeito dinâmico positivo na reprodução, desde que a ovelha se encontre em ECC intermediários no início da suplementação (Mellado et al., 2004; Cezar e Sousa, 2006).

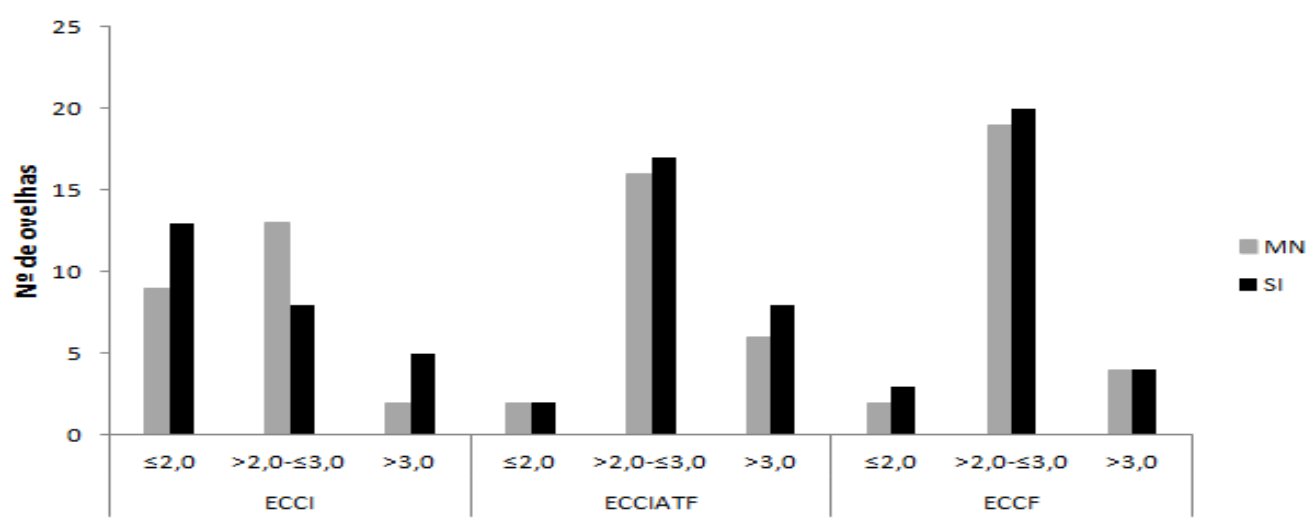

Figura 2. Número de ovelhas Morada Nova (MN) e Santa Inês (SI) em cada classe de escore da condição corporal no início do flushing (ECCI), no momento da IATF (ECCIATF) e no final do flushing (ECCF). 
O protocolo à base de esponja de progestágeno, eCG e cloprostenol foi eficiente para induzir o estro em ovelhas MN e SI, sendo que $88,2 \%$ das ovelhas apresentaram estro em até 48 horas após a retirada de esponja MAP. Resultados semelhantes foram encontrados por Santos et al. (2011), que utilizaram os mesmos hormônios para a sincronização de estro em ovelhas mestiças lanadas e deslanadas.
Houve diferença significativa quanto ao início de manifestação de estro entre as ovelhas MN e SI ( $<<0,01$ ), sendo que $37,5 \%$ das ovelhas $\mathrm{MN}$ apresentaram estro 24 horas após a retirada da esponja, enquanto $55,6 \%$ das ovelhas SI apresentavam comportamento de estro 36 horas após a retirada da esponja (Fig. 3).

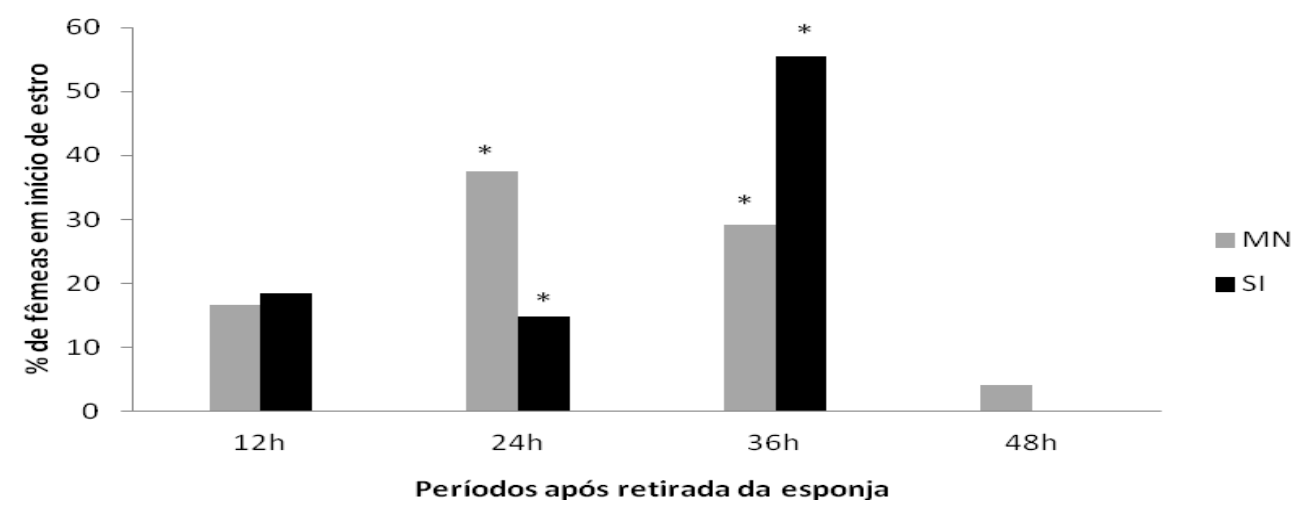

Figura 3. Porcentagem de ovelhas Morada Nova (MN) e Santa Inês (SI) em início de estro após 12, 24, 36 e 48 horas da retirada de esponja de progestágeno (MAP) e aplicação de eCG e cloprostenol para sincronização de estro. * Diferença significativa entre as raças (MN x SI) em cada período (teste de Fisher, $5 \%)$.

Segundo Dias et al. (2001), a maior concentração de estro de ovelhas deslanadas se dá 48 horas após a retirada da esponja e administração de eCG. No entanto, no presente estudo, $20 \%$ das ovelhas apresentaram estro até 24 horas após retirada da esponja. Alguns trabalhos demonstram que protocolos que utilizam eCG na retirada da esponja adiantam o início do estro (Dias et al., 2001), o que pode ser explicado pelo fato de o eCG aumentar a concentração de estrógeno em decorrência do efeito estimulatório sobre o crescimento de folículos. Como consequências, as elevadas concentrações de estrógeno induzem o comportamento de estro e a ocorrência do pico pré-ovulatório de LH (Rodrigues et al., 2004). Assim, as manifestações de estro que ocorreram antes das 48 horas da retirada da esponja podem ser explicadas pela aplicação de eCG.

Observou-se, no presente trabalho, que houve variação individual do tempo de início do estro entre as ovelhas (Fig. 3), o que mostra pouca eficiência do protocolo para sincronização de estro em ovelhas deslanadas. Segundo Menchaca et al. (2004), o uso de prostaglandina aumenta a variabilidade do tempo de início de manifestação do estro, pois o efeito da prostaglandina na luteólise depende do estágio de maturação do corpo lúteo e sua sensibilidade a esta, que é variável entre animais em diferentes fases do ciclo estral.

A taxa de prenhez obtida após sincronização de estro e IATF foi de $31,37 \%$ (Tab. 2), não diferindo entre as raças (MN e SI). Em estudos semelhantes, Dias et al. (2001) e Machado et al. (2006) obtiveram taxa média de prenhez de $30 \%$. Porém, Padilha et al. (2011) relataram taxa de prenhez de até $80 \%$ após realização de IATF em ovelhas deslanadas. Essa variação entre os estudos pode ser explicada pela utilização de diferentes tipos de protocolos de sincronização de estro, raças e condições nutricionais das fêmeas. Além disso, o resultado da inseminação artificial em ovinos é influenciado pela variação individual da anatomia da cérvice uterina, o que dificulta a deposição cervical do sêmen (Machado et al., 2006). 
Efeito do flushing...

Tabela 2. Taxas de prenhez por IATF (PIATF), de retorno ao estro (RETE), de prenhez após repasse (PREP) e de prenhez total (PT) durante a estação reprodutiva de ovelhas Morada Nova (MN) e Santa Inês (SI)

\begin{tabular}{l|cccc} 
Raça & $\begin{array}{c}\text { PIATF } \\
\%\left(\mathrm{n}^{\circ} / \mathrm{n}^{\circ} \text { total }\right)\end{array}$ & $\begin{array}{c}\text { RETE } \\
\left(\mathrm{n}^{\circ} / \mathrm{n}^{\circ} \text { total }\right)\end{array}$ & $\begin{array}{c}\text { PREP } \\
\%\left(\mathrm{n}^{\circ} / \mathrm{n}^{\circ} \text { total }\right)\end{array}$ & $\begin{array}{c}\text { PT } \\
\%\left(\mathrm{n}^{\circ} / \mathrm{n}^{\circ} \text { total }\right)\end{array}$ \\
\hline MN & $33,3(8 / 24) \mathrm{a}$ & $20,8(5 / 24) \mathrm{b}$ & $37,5(6 / 16) \mathrm{a}$ & $58,3(14 / 24) \mathrm{a}$ \\
SI & $29,6(8 / 27) \mathrm{a}$ & $55,6(15 / 27) \mathrm{a}$ & $68,4(13 / 19) \mathrm{a}$ & $77,8(21 / 27) \mathrm{a}$ \\
\hline TOTAL & $31,4(16 / 51)$ & $39,3(20 / 51)$ & $55,3(19 / 35)$ & $68,6(35 / 51)$ \\
\hline
\end{tabular}

${ }^{\mathrm{a}}$ Médias seguidas de letras iguais não diferem nas colunas (teste de Fisher, $5 \%$ ).

Apesar da taxa de prenhez observada no presente trabalho ser semelhante à encontrada por outros autores (Dias et al.,2001; Machado et al., 2006), esta não é considerada satisfatória para um programa de sincronização de estro e IATF, já que, segundo Bicudo (2003), a massificação da IATF como prática de manejo reprodutivo está na dependência do uso de uma única IATF com resultado mínimo de $60,0 \%$ de fertilidade ao parto.

Em ovelhas, o estro possui duração de aproximadamente 24 a 30 horas, sendo que a ovulação ocorre em seu terço final e o oócito tem viabilidade apenas de 12 a 24 horas após a ovulação (Godfrey et al., 1999). Considerando-se que as ovelhas apresentaram início de comportamento de estro antes de 24 horas após a retirada da esponja (Fig. 3), a IATF realizada aproximadamente 55 horas após a retirada da esponja MAP, conforme recomendado por Menchacas e Rubianes (2004), foi tardia. Ou seja, a manifestação de estro anteriormente ao momento esperado pode ter atrapalhado a fecundação após inseminação, o que ocasionou a baixa taxa de prenhez.

A taxa de retorno ao estro após a IATF foi maior $(\mathrm{p}<0,05)$ no grupo de ovelhas SI $(55,6 \%)$ do que no grupo de ovelhas MN (20,8\%). Por outro lado, a taxa de prenhez do repasse não foi estatisticamente diferente $(\mathrm{p}>0,05)$ entre as raças (Tab. 2).

A taxa de prenhez após repasse e de prenhez total foram semelhantes às relatadas por Martinez-Tinajero (2008). Porém, Santos (2011) obteve taxa de prenhez de $90 \%$ em estação reprodutiva com monta natural após sincronização de estro, utilizando 8 machos para 48 fêmeas. Dessa forma, pode-se supor que a baixa taxa de prenhez encontrada no presente trabalho seja decorrente da utilização de apenas um reprodutor para várias ovelhas que retornaram ao estro em um mesmo momento. Porém, outros efeitos, tais como condições climáticas, nutricionais, ou mesmo de manejo, podem ter influenciado nos resultados.

Em ovelhas deslanadas, a condição corporal da fêmea reflete diretamente nos resultados da sincronização de estro com protocolos hormonais e IATF (Martin et al., 2004), sendo a nutrição o principal fator que interfere na estacionalidade reprodutiva (Santos et al., 2011). Dessa forma, foi avaliado o efeito da classe de ECC (CECC) das ovelhas sobre as taxas de manifestação de estro e de prenhez (Tab. 3).

Tabela 3. Taxa de estro (ESTRO), de prenhez por IATF (PIATF) e prenhez após monta natural no repasse (PREP) de acordo com a classe de ECC (CECC) no momento da sincronização, da realização da IATF e do repasse

\begin{tabular}{l|ccc}
\multirow{2}{*}{ CECC } & $\begin{array}{c}\text { ESTRO } \\
\left(\mathrm{n}^{\circ} / \mathrm{n}^{\circ} \text { total }\right)\end{array}$ & $\begin{array}{c}\text { PIATF } \\
\%\left(\mathrm{n}^{\circ} / \mathrm{n}^{\circ} \text { total }\right)\end{array}$ & $\begin{array}{c}\text { PREP } \\
\%\left(\mathrm{n}^{\circ} / \mathrm{n}^{\circ} \text { total }\right)\end{array}$ \\
\hline$\leq 2,0$ & $80,00(4 / 5) \mathrm{a}$ & $40,00(2 / 5) \mathrm{a}$ & $0,00(0 / 7) \mathrm{b}$ \\
$>2,0 \leq 3,0$ & $93,75(30 / 32) \mathrm{a}$ & $40,63(13 / 32) \mathrm{a}$ & $58,33(14 / 24) \mathrm{a}$ \\
$>3,0$ & $78,57(11 / 14) \mathrm{a}$ & $7,14(1 / 14) \mathrm{b}$ & $100,00(4 / 4) \mathrm{a}$ \\
\hline
\end{tabular}

Médias seguidas de letras iguais não diferem entre as classes de escore da condição corporal (teste de Fisher, 5\%). 
A CEEC não influenciou na taxa de manifestação de estro $(p>0,05)$, mas interferiu nas taxas de prenhez $(\mathrm{p}<0,05)$, tal como relatado por Ribeiro (2003). Na IATF obteve-se maior taxa de prenhez nas ovelhas que apresentavam ECC até 3,0. Por outro lado, observou-se maior taxa de prenhez no repasse de animais com ECC acima de 2,5 $(\mathrm{p}<0,05)$.

Diversos trabalhos já demonstraram o efeito positivo da suplementação de ovelhas durante a estação reprodutiva (Catunda, 2011; Santos et al., 2011). Todavia, está bem descrito que a suplementação é positiva apenas para ovelhas que apresentam baixo ECC no início da estação reprodutiva, mais especificamente aquelas que apresentam ECC entre 1,5 a 3,0 (Boucinhas et $a l ., 2006)$, quando o efeito que a nutrição exerce sobre a reprodução se dá de forma dinâmica, através do ganho compensatório. Esse fato explica a maior taxa de prenhez por IATF nos animais com ECC até 3,0, sendo que esses animais tiveram ainda a possibilidade de ganhar peso por meio da suplementação nas demais quatro semanas de suplementação da estação reprodutiva. Possivelmente o flushing tenha exercido efeito positivo, o que garantiu o estabelecimento da prenhez, como um efeito dinâmico da suplementação na reprodução. Além disso, ovelhas com ECC maior que 3,0 no momento da concepção, além de não responderem mais ao flushing, possuem maiores chances de perdas embrionárias (Robinson et al., 2002).

Por outro lado, Ribeiro (2003) considera que o máximo desempenho reprodutivo é alcançado por animais que atingem ECC entre 3,0 e 3,5 no final da estação reprodutiva, o que está em concordância com o observado após o repasse no presente trabalho (Tab. 3).

De qualquer forma, o efeito mais importante foi devido à variação de peso que o animal sofreu durante a estação reprodutiva e, por isso, o manejo alimentar de ovelhas durante esse período deve ser criteriosamente acompanhado para que estejam com ECC o mais próximo possível de 3,0 no momento da cobertura. Sendo assim, orienta-se a utilização do flushing em animais com ECC entre 2,0 ou 2,5, com a manutenção de oferta de energia a partir da condição corporal de 3,0 , pois, se o consumo energético for inferior à demanda do animal, o ECC piora e leva a respostas de efeitos dinâmicos negativos na fertilidade (Cezar e Sousa, 2006). Em vista disso, os sete animais do presente estudo que apresentaram ECC menor que 2,0 durante o repasse perderam peso ou mantiveram baixo ECC (Tab. 3), o que refletiu na ausência de prenhez mesmo após o repasse com monta natural.

Com relação à porcentagem de partos múltiplos obtidos na estação reprodutiva, foi observado que a raça e a classe de ECC não interferiram significativamente nessa variável (Fig. 4). Embora trabalhos anteriores tenham demonstrado que ECC maior que 3,0 resultam em maiores taxas de ovulação (Robinson, 2002; Viñoles et al., 2005), esse efeito não foi verificado no presente trabalho, provavelmente devido à pouca quantidade de animais com ECC $\leq 2,0$ e $>3,0$.
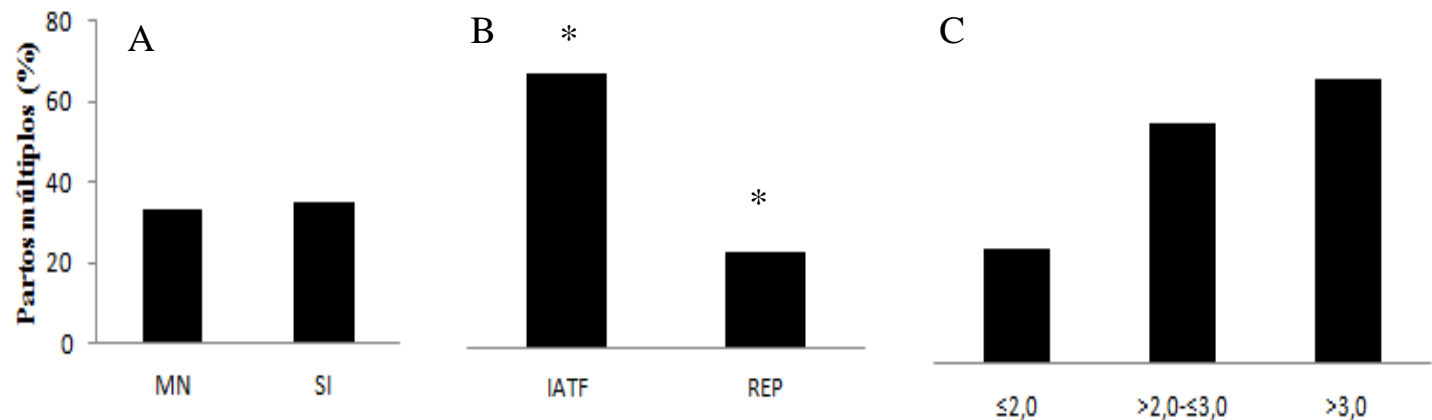

Figura 4. Partos múltiplos de acordo com a raça (A), origem da prenhez (IATF ou Repasse) (B) e classe de ECC $(\leq 2 ;>2 \leq 3 ;>3)(\mathrm{C})$. *Diferença significativa entre origem da prenhez (teste de Fisher, $5 \%$ ). 
Por outro lado, obteve-se maior porcentagem de partos múltiplos em ovelhas prenhes por IATF do que em ovelhas prenhes após repasse $(\mathrm{p}>0,05)$. Provavelmente esse efeito ocorreu pela aplicação de eCG, pois já foi demonstrado que sua utilização em protocolo hormonal para sincronização de estro induz ovulações de mais de um folículo pré-ovulatório, devido aos efeito semelhantes ao LH (Barret et al., 2004).

\section{CONCLUSÕES}

O flushing resultou em ganho de peso e aumento do ECC, o que garantiu a padronização do rebanho para a estação reprodutiva e, consequentemente, acarretou em melhor desempenho reprodutivo. $\mathrm{O}$ protocolo de sincronização de estro utilizado foi eficiente na indução de estro, porém adiantou o início do comportamento de estro e não foi eficiente para sincronização de ovelhas deslanadas.

\section{REFERÊNCIAS}

ANDRADE JÚNIOR, A.S.; BASTOR, E.A.; BARROS, A.H.C. et al. Classificação climática e regionalização do semi-árido do Estado do Piauí sob cenários pluviométricos distintos. Rev. Cienc. Agron., v.36, p.143-151, 2005.

ANUÁRIO da pecuária brasileira. São Paulo: FNP, 2012, 378p.

BARRET, D.M.W.; BARTLEWSKI, P.M.; BATISTA-ARTEAGA, M. et al. Ultrasound and endocrine evaluation of the ovarian response to a single dose of $500 \mathrm{IU}$ of eCG following a 12-day treatment with progestogen-releasing intravaginal sponges in the breeding and nonbreeding seasons in ewes. Theriogenology, v.61, p.311-327, 2004.

BICUDO, S.D.; SOUSA, D.B.; TAKADA, L. Possibilidades e limitações da inseminação com sêmen ovino refrigerado. Rev. Bras. Rep. Anim., v.27, p.120-127, 2003.

BOUCINHAS, C.C.; SIQUEIRA, E.R.; MAESTÁ, S.A. Dinâmica do peso e da condição corporal e eficiência reprodutiva de ovelhas da raça Santa Inês e mestiças Santa Inês-Suffolk submetidas a dois sistemas de alimentação em intervalos entre partos de oito meses. Cienc. Rural, v.36, p.904909, 2006.
CATUNDA, A.G.V. Avaliação dos parâmetros fisiológicos, metabólicos e reprodutivos de ovelhas deslanadas submetidas à suplementação energética criadas em sistema semi-intensivo no Nordeste do Brasil. 2011. 109p. Tese (Doutorado em reprodução Animal) - Universidade Estadual do Ceará, Fortaleza.

CEZAR, M.F.; SOUSA, W.H. Avaliação e utilização da condição corporal como ferramenta de melhoria da reprodução e produção de ovinos e caprinos de corte. Rev. Bras. Zootec., v.35, p.649-678, 2006.

DIAS, F.E.F.; LOPES JÚNIOR, E.S.; VILLAROEL, A.B.S. et al. Sincronização do estro, indução da ovulação e fertilidade de ovelhas deslanadas após tratamento hormonal com gonadotrofina coriônica equina. Arq. Bras. Med. Vet. Zootec., v.53, p.618-623, 2001.

GODFREY, R.W.; COLLINS, J.R.; HENSLEY, E.L.; WHEATON, J.E. Estrus synchronization and artificial insemination of hair sheep ewes in the tropics. Theriogenology, v.51, p.985-997, 1999.

MACHADO, V.P.; NUNE, J.F.; ARAÚJO, A.A. et al. Fertilidade após inseminação intracervical ou laparoscópica intrauterina de ovelhas utilizando diluidores a base de coco. Braz. J. Vet. Res. Anim. Sci., v.43, p.43-49, 2006.

MARTIN, G.B.; RODGER, J.; BLACHE, D. Nutritional and environmental effects on

reproduction in small ruminants. Reprod. Fertil. Dev., v.16, p.491-501, 2004.

MARTINEZ-TINAJERO, J.J.; TORRESESQUERDA, M.T.S.; TORRESHERNÁNDES, G. et al. Compotament reproductivo de ovejas F1 (Dara x Merino) sincronizadas com CIDR y dos tempos de aplicacíon de GnRH. Univ. y Ciencia, v.24, p.175-182, 2008.

MELLADO, M.; VALDEZ, R.; LARA, L.M. et $a l$. Risk factors involved in conception, abortion, and kidding rates of goats under extensive conditions. Small Rum. Res., v.55, p.191-198, 2004.

MENCHACA, A.; RUBIANES, E. New treatments associated with timed artificial insemination in small ruminants. Reprod. Fertil. Dev, v.16, p.403-413, 2004. 
MORI, R.M.; RIBEIRO, E.L.A.; MIZUBUTI, I.Y. et al. Desempenho reprodutivo de ovelhas submetidas a diferentes formas de suplementação alimentar antes e durante a estação de monta. Rev. Bras. Zootec., v.35, p.1122-1128, 2006.

NUTRIENT Requirements of Small RuminantsNRC: Sheep, Goats, Cervids, and New World Camelids. Washington: National Academy of Science, 2007. 347p.

PADILHA, R.T; MAGALHÃES, D.M.; MAIAJÚNIOR, A. et al. Efeito de diferentes dispositivos intravaginais na sincronização estral e taxa de gestação em ovelhas deslanadas submetidas a IATF via cervical superficial com sêmen refrigerado. Rev. Bras. Cienc. Agrar., v.3, p.538-543, 2011.

RIBEIRO, L.A.O. Relação entre condição corporal e a idade das ovelhas no encarneiramento com a prenhez. Cienc. Rural, v.33, p.357-361, 2003.

ROBINSON, J.J. Nutrition and reproduction. Anim. Reprod. Sci., v.42, p.25-34, 2002.
RODRIGUES, L.F.S.; ARAUJO, A.A.; NUNES, J.F. et al. Sincronização do estro em ovelhas deslanadas: efeito de diferentes doses de gonadotrofina coriônica equina sobre a taxa de ovulação. Rev. Cienc. Agrar., v.41, p.215-222, 2004.

SANTOS, G.M.G.; SILVA-SANTOS, K.C.; MELO-STERZA F.A. et al. Desempenho reprodutivo de ovelhas mestiças lanadas e deslanadas submetidas a protocolo hormonal a base de progestágeno e eCG durante a contraestação reprodutiva. Semina: Cienc. Agrar., v.32, p.723-732, 2011.

THOMPSON, J.; MEYER, H. Body condition scoring sheep. Oregon: Oregon State University, 1994, 4p.

VIÑOLES, C.; FORSBERG, M.; MARTIN, G.B. et al. Short term nutricional supplementation of ewes in low body conditions affects follicle development due to an increase in glucose and metabolic hormones. Reproduction, v.129, p.299-309, 2005. 